\title{
A GROUPOID FORMULATION OF THE BAIRE CATEGORY THEOREM
}

\author{
JONATHAN BROWN AND LISA ORLOFF CLARK
}

\begin{abstract}
We prove that the Baire Category Theorem is equivalent to the following: Let $G$ be a topological groupoid such that the unit space is a complete metric space, and there is a countable cover of $G$ by neighbourhood bisections. If $G$ is effective, then $G$ is topologically principal.
\end{abstract}

\section{INTRODUCTION}

Theorem 1.1 (The Baire Category Theorem). (See, for example, [6, Theorem 7.7.2].) Suppose $X$ is a complete metric space. If $\left\{C_{n}\right\}$ is a countable collection of closed subsets of $X$, each with empty interior, then $\bigcup_{n} C_{n}$ has empty interior.

The proof of the Baire Category Theorem, originally formulated by Baire in the 1890's, requires a variant of the Axiom of Choice [3, Chapter 13]. In fact, [1] and [4] show the Baire Category Theorem is equivalent to the the Principle of Dependant Choices which says:

Suppose $X$ is a set and $R \subseteq X \times X$ is a relation such that for each $x \in X$, there exists $y \in X$ such that $(x, y) \in R$. Then there is a sequence $\left\{x_{n}\right\} \subseteq X$ such that $\left(x_{n}, x_{n+1}\right) \in R$ for all $n$.

The Principle of Dependent Choices falls strictly between the Countable Axiom of Choice and the Axiom of Choice; see 5 ] for more details.

In this note, we show the Baire Category Theorem is equivalent to Theorem 3.4 below, which is a surprising result about effective groupoids. We discovered a version of Theorem 3.4 in our study of simple groupoid $C^{*}$-algebras [2, Lemma 3.3]. A version also appears in [8, Proposition 3.6] in the context of maximal abelian subalgebras of $C^{*}$ algebras. That Theorem 3.4 implies the Baire Category Theorem is entirely new; but even our proof that the Baire Category Theorem implies Theorem 3.4 is different from those in [2] and [8].

\section{Preliminaries}

A groupoid $G$ is a small category in which every morphism is invertible. We identify the set of objects of $G$ with the set of identity morphisms and denote this set $G^{(0)}$. For $\gamma \in G$, we denote the range and source (domain) of $\gamma$ by $r(\gamma)$ and $s(\gamma)$ respectively. Thus $r, s: G \rightarrow G^{(0)} \subseteq G$. We define $G^{(2)}:=\{(\gamma, \eta) \in G \times G: r(\eta)=s(\gamma)\} ; G^{(2)}$ consists

Date: September 12, 2012.

2000 Mathematics Subject Classification. 03E25, 54E52, 22A22.

Key words and phrases. Baire Category Theorem, topologically principal groupoid, effective groupoid, Principle of Dependent Choices. 
of precisely those pairs of morphisms that can be composed in $G$. For any $x \in G^{(0)}$, the isotropy group at $x$ is the group

$$
x G x:=\{\gamma \in G: r(\gamma)=s(\gamma)=x\} .
$$

The isotropy subgroupoid of $G$ is

$$
\operatorname{Iso}(G):=\bigcup_{x \in G^{(0)}} x G x
$$

which is itself a groupoid. If $B \subseteq G$, then we also write $\operatorname{Iso}(B):=\operatorname{Iso}(G) \cap B$. We say $G$ is a group bundle if $\operatorname{Iso}(G)=G$.

A groupoid $G$ is a topological groupoid if $G$ is equipped with a topology so that composition and inversion are continuous. In this case, $r$ and $s$ are continuous maps. If $G^{(0)}$ is Hausdorff, then the continuity of $r$ and $s$ implies that $\operatorname{Iso}(G)$ is a closed subset of $G$.

An open set $A \subseteq G$ is called an open bisection if $r(A)$ and $s(A)$ are open in $G$ and $r$ and $s$ restricted to $A$ are homeomorphisms onto their image; in particular $r$ and $s$ are injective on $A$.

We say a groupoid $G$ is topologically principal if the set $\left\{x \in G^{(0)}: x G x \neq\{x\}\right\}$ has empty interior in $G^{(0)}$. A groupoid $G$ is effective if $\operatorname{Iso}(G)-G^{(0)}$ has empty interior.

\section{WHEN DOES EFFECTIVE IMPLY TOPOLOGICALLY PRINCIPAL?}

In [8, Proposition 3.6(ii)] Renault considers effective groupoids whose unit spaces are 'Baire'. We can interpret Renault's result as saying that Theorem 1.1 implies the following:

Theorem 3.1. [8, Proposition 3.6(ii)] Suppose G is a topological groupoid such that:

(1) the unit space is a complete metric space;

(2) $G$ has a countable cover consisting of open bisections.

If $G$ is effective, then $G$ is topologically principal.

Our original intention was to show that Theorem 3.1 is equivalent to Theorem 1.1 . However, we eventually realised that such a result will only hold if we weaken the hypotheses of Theorem 3.1. To see why, consider the class of effective groupoids constructed in Example 3.2 below. Each has the property that Theorem 1.1 implies it is topologically principal. At the same time, groupoids in this class may not satisfy the hypotheses of Theorem 3.1. (We will also use this class of examples later in the proof of our main result.)

Example 3.2. Let $X$ be a complete metric space and $\left\{C_{n}\right\}$ be a countable collection of closed subsets of $X$, each with empty interior. Define $C:=\bigcup_{n} C_{n}$.

Let $G$ be the group bundle with unit space $X$ and isotropy groups

$$
x G x:= \begin{cases}\mathbb{Z}_{2}, & \text { if } x \in C ; \\ \{0\}, & \text { otherwise }\end{cases}
$$

We identify the identity element $0 \in x G x$ with $x$. For each $x \in C$, we write $\gamma_{x}$ for the nontrivial element of $x G x$. Notice that

$$
G^{(2)}=\{(x, x): x \in X\} \cup\left\{\left(\gamma_{x}, \gamma_{x}\right): x \in C\right\} \cup\left\{\left(x, \gamma_{x}\right): x \in C\right\} \cup\left\{\left(\gamma_{x}, x\right): x \in C\right\} .
$$


To make $G$ into a topological groupoid, first let $\mathcal{T}$ be the topology for $X$. Define the collection

$$
\mathcal{B}:=\mathcal{T} \cup\left\{V \subseteq G: V=(W-\{x\}) \cup\left\{\gamma_{x}\right\} \text { for some } W \in \mathcal{T} \text { and } x \in C \cap W\right\} .
$$

The collection $\mathcal{B}$ forms a basis for a topology on $G$. To see this, note that $\mathcal{B}$ covers $G$ and since $\mathcal{T}$ is the topology for $X$, it is easy to see that $U, V \in \mathcal{B}$ implies $U \cap V \in \mathcal{B}$. We claim that $G$ endowed with the topology generated by $\mathcal{B}$ is a topological groupoid. Indeed, inversion is given by the identity and is thus continuous. Now let $m: G^{(2)} \rightarrow G$ be the composition map. Fix $V \in \mathcal{B}$. If $V \in \mathcal{T}$, then

$$
m^{-1}(V)=\{(x, x): x \in V\} \cup\left\{\left(\gamma_{x}, \gamma_{x}\right): x \in V\right\} .
$$

If $V=(W-\{y\}) \cup\left\{\gamma_{y}\right\}$ for some $W \in \mathcal{T}$ and $y \in C \cap W$, then

$$
m^{-1}(V)=\{(x, x): x \in W-\{y\}\} \cup\left\{\left(\gamma_{y}, y\right),\left(y, \gamma_{y}\right)\right\} \cup\left\{\left(\gamma_{x}, \gamma_{x}\right): x \in(W-\{y\}) \cap C\right\} .
$$

In both cases, it is straightforward to show that $m^{-1}(V)$ is open in $G^{(2)}$, hence composition is continuous as claimed.

Since every element of $\mathcal{B}$ intersects the unit space, the set $\operatorname{Iso}(G)-G^{(0)}=G-G^{(0)}$ contains no open sets, so $G$ is effective. By construction, $G^{(0)}=X$ is a complete metric space and

$$
C=\left\{x \in G^{(0)}: x G x \neq\{x\}\right\},
$$

so Theorem 1.1 implies that $G$ is also topologically principal. Notice that $G$ need not satisfy item (2) of Renault's Theorem 3.1. Indeed if $X=[0,1]$ and $C_{n}=C$ is the Cantor set for all $n$, there is no countable cover of $G$ consisting of open bisections. To see this suppose $\left\{U_{i}\right\}$ is any countable open cover of $G$. Since the Cantor set is uncountable there exists an $i_{0}$ such that $A_{i_{0}}:=\left\{x \in C: \gamma_{x} \in U_{i_{0}}\right\}$ is uncountable. For each $x \in A_{i_{0}}$ pick a basis element $\left(V_{x}-\{x\}\right) \cup\left\{\gamma_{x}\right\}$ contained in $U_{i_{0}}$. Since the standard basis for $[0,1]$ is given by connected intervals, we can assume that $V_{x}$ is connected. For each $n \in \mathbb{Z}^{+}$define $D_{n}:=\left\{x \in A_{i_{0}}:\right.$ diameter of $V_{x}$ is greater than $\left.2 / n\right\}$. Since $A_{i_{0}}$ is uncountable, there exists $n_{0}$ such that $D_{n_{0}}$ is uncountable. Now consider the partition $\left\{P_{m}:=\left[m / 2 n_{0},(m+1) / 2 n_{0}\right]\right\}$ of $[0,1]$ where $0 \leq m \leq 2 n_{0}-1$. Since $D_{n_{0}}$ is uncountable, there exists an $m_{0}$ such that $D_{n_{0}} \cap P_{m_{0}}$ is uncountable. By the definition of $D_{n_{0}}$ this implies that for every $x \in D_{n_{0}} \cap P_{m_{0}}$ both $x$ and $\gamma_{x}$ are in $U_{i_{0}}$ and so $U_{i_{0}}$ is not an open bisection.

While the groupoids considered above need not have a countable cover of open bisections, they do have a countable cover consisting of 'well-behaved sets'. We call these sets neighbourhood bisections. (We denote the interior of a set $D$ by $\operatorname{Int}(D)$.)

Definition 3.3. A set $B \subseteq G$ is called a neighbourhood bisection if the following hold:

(1) $B \subseteq \overline{\operatorname{Int}(B)}$;

(2) $\left.r\right|_{B}$ and $\left.s\right|_{B}$ are injective;

(3) $r(B)$ and $s(B)$ are open in $G$;

(4) $\operatorname{Int}(B)$ is an open bisection;

(5) $B-\operatorname{Int}(B) \subseteq \operatorname{Iso}(B)-G^{(0)}$.

In the next section we prove the following theorem is equivalent to Theorem 1.1. One part of our proof involves showing the class of groupoids constructed in Example 3.2 do indeed have a countable cover consisting of neighbourhood bisections. 
Theorem 3.4. Suppose $G$ is a topological groupoid such that:

(1) the unit space is a complete metric space;

(2) G has a countable cover consisting of neighbourhood bisections.

If $G$ is effective, then $G$ is topologically principal.

Remark 3.5. Suppose $G$ is a groupoid satisfying the hypotheses of Theorem 3.4, then $G^{(0)}$ is open in $G$. To see this, let $\left\{B_{n}\right\}$ be a countable cover of $G$ by neighbourhood bisections, then $G^{(0)}=\bigcup r\left(B_{n}\right)$ which is open.

Remark 3.6. An étale groupoid is a topological groupoid that has a cover consisting of open bisections. When studying $C^{*}$-algebras associated to groupoids, one often considers second-countable, locally compact, Hausdorff groupoids that are étale. These groupoids satisfy the hypothesis of Theorem 3.4.

Remark 3.7. Suppose $G$ is a topological groupoid. If $r$ is an open map, then $G$ topologically principal implies $G$ is effective. See [2, Examples 6.3 and 6.4] for examples of groupoids (that do not satisfy the hypothesis of Theorem 3.4) that are effective but not topologically principal.

\section{Main Result}

Theorem 4.1. Theorem 1.1 is equivalent to Theorem 3.4.

Before we prove Theorem 4.1, we establish the following two lemmas. The first lemma is used to prove the second (Lemma 4.3); Lemma 4.3 is a key step in our proof of Theorem 4.1 .

Lemma 4.2. Suppose $G$ is a topological groupoid such that $G^{(0)}$ is open in $G, B \subseteq G$ is a neighbourhood bisection and $D \subseteq B$ is closed in $B$ where $B$ is endowed with the subspace topology. Suppose that $B-\operatorname{Int}(B) \subseteq D$. Then $r(D)$ is closed in $r(B)$ where $r(B)$ is endowed with the subspace topology.

Proof. Let $G, B$ and $D$ be as stated. Then

$$
D=(D \cap \operatorname{Int}(B)) \cup(B-\operatorname{Int}(B))
$$

which means

$$
r(D)=r(D \cap \operatorname{Int}(B)) \cup r(B-\operatorname{Int}(B)) .
$$

Since $\left.r\right|_{B}$ is a bijection onto its image, $r(B-\operatorname{Int}(B))=r(B)-r(\operatorname{Int}(B))$ which is closed in $r(B)$ as $r(\operatorname{Int}(B))$ is open. Further $r(D \cap \operatorname{Int}(B))$ is closed in $r(\operatorname{Int}(B))$ because $r$ restricted to $\operatorname{Int}(B)$ is a homeomorphism. Thus there exists a closed set $C$ such that $r(\operatorname{Int}(B)) \cap C=r(\operatorname{Int}(B) \cap D)$. Therefore

$$
\begin{aligned}
r(D) & =r(\operatorname{Int}(B) \cap D) \cup r(B-\operatorname{Int}(B)) \\
& =(r(\operatorname{Int}(B)) \cap C) \cup(r(B-\operatorname{Int}(B)) \cap C) \cup r(B-\operatorname{Int}(B)) \\
& =(r(B) \cap C) \cup r(B-\operatorname{Int}(B))
\end{aligned}
$$

which is closed in $r(B)$. 
Lemma 4.3. Suppose $G$ is an effective groupoid such that $G^{(0)}$ is open in $G$ and $B$ is a neighbourhood bisection. Then

(1) $r\left(\operatorname{Iso}(B)-G^{(0)}\right)$ has empty interior, and

(2) $\overline{r\left(\operatorname{Iso}(B)-G^{(0)}\right)}$ has empty interior.

Proof. For (1), by way of contradiction, suppose there exists a nonempty open set $W \subseteq$ $r\left(\operatorname{Iso}(B)-G^{(0)}\right)$. Thus $W \cap r(B) \neq \emptyset$, and since $B \subseteq \overline{\operatorname{Int}(B)}$, we have $W \cap r(\overline{\operatorname{Int}(B)}) \neq \emptyset$. Therefore

$$
W \cap \overline{r(\operatorname{Int}(B))} \neq \emptyset \text { because } r(\overline{\operatorname{Int}(B)}) \subseteq \overline{r(\operatorname{Int}(B))} .
$$

Hence $W \cap r(\operatorname{Int}(B))$ is a nonempty open set contained in $G^{(0)}$. Since

$$
\phi:=\left.r\right|_{\operatorname{Int}(B)}
$$

is a homeomorphism,

$$
\phi^{-1}(W \cap r(\operatorname{Int}(B)))
$$

is a nonempty open subset of $\operatorname{Int}(B)$ and thus is open in $G$. Since $r$ is injective on $B$ and $W \subseteq r\left(\operatorname{Iso}(B)-G^{(0)}\right)$,

$$
\left(\phi^{-1}(W \cap r(\operatorname{Int}(B))) \subseteq \operatorname{Iso}(B)-G^{(0)} \subseteq \operatorname{Iso}(G)-G^{(0)} .\right.
$$

This is a contradiction because $G$ is effective.

For (2), by way of contradiction, assume there exists a nonempty open subset

$$
V \subseteq \overline{r\left(\operatorname{Iso}(B)-G^{(0)}\right)}
$$

Notice that $V \cap r(B)$ is a nonempty open subset of $G^{(0)}$. Further,

$$
V \cap r(B) \subseteq \overline{r\left(\operatorname{Iso}(B)-G^{(0)}\right)} \cap r(B) .
$$

We show $\overline{r\left(\operatorname{Iso}(B)-G^{(0)}\right)} \cap r(B)=r\left(\operatorname{Iso}(B)-G^{(0)}\right)$. Since $\operatorname{Iso}(B)$ is closed in $B$ and $G^{(0)}$ is open, $\operatorname{Iso}(B)-G^{(0)}$ is also closed in $B$. Also, $B-\operatorname{Int}(B) \subseteq \operatorname{Iso}(B)-G^{(0)}$ by assumption. Therefore we apply Lemma 4.2 to see that $r\left(\operatorname{Iso}(B)-G^{(0)}\right)$ is closed in $r(B)$. Thus

$$
\begin{aligned}
& r\left(\operatorname{Iso}(B)-G^{(0)}\right)=\overline{r\left(\operatorname{Iso}(B)-G^{(0)}\right)} \cap r(B), \text { and so } \\
& V \cap r(B) \subseteq r\left(\operatorname{Iso}(B)-G^{(0)}\right)
\end{aligned}
$$

which contradicts item (1).

Proof of Theorem 4.1: Suppose Theorem 1.1 holds. Let $G$ be a topological groupoid with a countable cover of neighbourhood bisections $\left\{B_{n}\right\}$ such that $G^{(0)}$ is a complete metric space. Suppose also that $G$ is effective.

By Lemma 4.3)(2), the set $\overline{r\left(\operatorname{Iso}\left(B_{n}\right)-G^{(0)}\right)}$ has empty interior for every $n$. Define $C_{n}:=\overline{r\left(\operatorname{Iso}\left(B_{n}\right)-G^{(0)}\right)} \cap G^{(0)}$ for each $n$. Notice that each $C_{n}$ is a closed subset of $G^{(0)}$. Because $G^{(0)}$ is open in $G$, each $C_{n}$ also has empty interior in $G^{(0)}$. Applying Theorem 1.1 (Baire Category Theorem) to the collection $\left\{C_{n}\right\}$ we see that

$$
C:=\bigcup_{n} C_{n}
$$

has empty interior. By construction, $C$ contains the units with nontrivial isotropy. Therefore, $G$ is topologically principal. 
Conversely, suppose that Theorem 3.4 holds. Let $X$ be a complete metric space with topology $\mathcal{T}$ and $\left\{C_{n}\right\}$ be a countable collection of closed subsets of $X$, each with empty interior. With out loss of generality we can assume $C_{0}=\emptyset$. Let $C=\bigcup_{n} C_{n}$. Define $G$ as in Example 3.2. Since $G^{(0)}=X$ as a topological space, $G$ satisfies (1) of Theorem 3.4.

For each $n$, define

$$
B_{n}:=\left(X-C_{n}\right) \cup\left\{\gamma_{x}: x \in C_{n}\right\} .
$$

We claim that each $B_{n}$ is a neighbourhood bisection. To prove this, we must check each of the items in Definition 3.3. To see (1), first note that $X-C_{n}$ is open in $G$ and contained in $B_{n}$. Thus $X-C_{n} \subseteq \operatorname{Int}\left(B_{n}\right)$. We show that $B_{n} \subseteq \overline{X-C_{n}} \subseteq \overline{\operatorname{Int}\left(B_{n}\right)}$. Consider $\gamma_{x}$ for some $x \in C_{n}$. For every $V \in \mathcal{B}$ with $\gamma_{x} \in V$ we have $V=W-\{x\} \cup\left\{\gamma_{x}\right\}$ where $W \in \mathcal{T}$ and $x \in W$. Now $V \cap\left(X-C_{n}\right)=W \cap\left(X-C_{n}\right)$ is nonempty because $C_{n}$ has empty interior. Therefore $\gamma_{x} \in \overline{X-C_{n}}$. Since $B_{n}=X-C_{n} \cup\left\{\gamma_{x}: x \in C_{n}\right\}$, we have $B_{n} \subseteq \overline{X-C_{n}}$. That $B_{n}$ satisfies item (2) is clear and $r\left(B_{n}\right)=X=s\left(B_{n}\right)$ is open in $G$ giving us item (3). Since $r(V)$ is in $\mathcal{T}$ for every $V \in \mathcal{B}, r=s$ is an open map and hence $\left.r\right|_{\operatorname{Int}\left(B_{n}\right)}=\left.s\right|_{\operatorname{Int}\left(B_{n}\right)}$ is a homeomorphism with open image giving item (4). Lastly, since $B_{n} \cap G^{(0)}=X-C_{n} \subseteq \operatorname{Int}\left(B_{n}\right)$ and $G=\operatorname{Iso}(G)$, we get item (15). Thus $\left\{B_{n}\right\}_{n}$ is a countable cover of $G$ by neighbourhood bisections and $G$ satisfies item (2) of Theorem 3.4 .

We showed in Example 3.2 that $G$ is effective. Therefore $G$ is topologically principal by Theorem 3.4. Thus

$$
C=\left\{x \in G^{(0)}: x G x \neq\{x\}\right\}
$$

has empty interior proving Theorem 1.1.

Acknowledgements. Thanks to François Dorais for the very helpful email correspondence.

\section{REFERENCES}

[1] C.E. Blair, The Baire Category Theorem implies the Principle of Dependent Choices, Bull. Acad. Polon. Sci. Sr. Sci. Math. Astronom. Phys. 25 (1977), 933-934.

[2] J.H. Brown, L.O. Clark, C. Farthing and A. Sims, Simplicity of algebras associated to étale groupoids, submitted. arXiv:1204.3127v1 [math.OA]

[3] W. Dunham, The calculus gallery: masterpieces from Newton to Lebesgue, Princeton University Press, Princeton, NJ, 2005.

[4] R. Goldblatt, On the role of the Baire Category Theorem and Dependent Choice in the foundations of logic, J. Symbolic Logic 50 (1985), 412-422.

[5] T.J. Jech, The Axiom of Choice, reprint, Dover Publications, Inc., Mineola, NY, 2008.

[6] J.R. Munkres, Topology, second edition, Prentice Hall, Upper Saddle River, 2000.

[7] A. Paterson, Groupoids, inverse semigroups, and their operator algebras, Birkhäuser Boston, Inc., Boston, MA, 1999.

[8] J. Renault, Cartan subalgebras in $C^{*}$-algebras, Irish Math. Soc. Bulletin 61 (2008), 29-63.

Lisa Orloff Clark, Department of Mathematics and Statistics, University of Otago, PO Box 56, Dunedin Dunedin 9054, New Zealand.

E-mail address: lclark@maths.otago.ac.nz

Jonathan Brown, Mathematics Department, Kansas State University, 138 Cardwell Hall, Manhattan, KS 66506-2602, USA.

E-mail address: brownjh@math.kansas.edu 